of the report is to dismiss the notion that CVD is primarily a disease of the elderly: it causes three times as many deaths in 39- to 69-year-olds as do infectious parasitic diseases combined. "What we have to drive across to the public is that the burden of CVD is affecting people during the working years of life," says Howson.

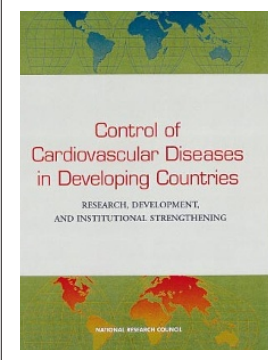

The report includes recommendations for areas of $R \& D$ focus, such as development of targeted prevention strategies, re duction of tobacco use, detection and treatment of high

blood pressure, pilot studies to evaluate the effects of low cost cardiovascular drugs, and the assessment of affordable clinical care for CVD.

Most importantly, the IOM says that it is essential that members of the developing countries themselves be involved in creating and running the proposed programs. "The west has a history of 'parachute' research-we drop in a country and then leave, taking the research with us. The people are left worse off than before. But there is a growing appreciation that to achieve global health we have to leave behind the hardware that will allow activity to continue and grow," says Howson.

A preliminary version of the report was presented in Geneva in June at the Global Forum for Health Research-an international organization of governments from low and middle income countries, health policy experts and researchers that meets to convince the more wealthy governments and other investors of the value of biomedical research in improving health and enhancing economic development among the poorest nations.

The Forum has al ready adopted the report's recommendation to form a steering committee to guide cardiovascular $R \& D$ efforts. The committee aims to establish a competitive research grant program and form a global health policy network for CVD. And in response to the reports findings, the National Institutes of Health will host a major conference next year to bring together interested parties to decide on funding mechanisms for the proposed areas of CVD R\&D.

\section{Kristine Novak, New York}

\title{
New rating system for UK universities
}

British medical schools are locked in a heated debate over how best to respond to suggested changes in the rating system that will be used in 2001 to assess the quality of their research-a key part of the process of deciding how much government money they should receive. At the heart of the dispute is whether, and if so how much, responsibility for drawing up the performance ratings of medical schools in hospitalbased clinical research should be allocated to subpanels of experts in particular fields.

In a discussion document released last month, the Higher Education Funding Council for England (HEFCE), which carries out the assessment exercise for all British universities at regular five-yearly intervals, outlines a possible model that will divide research into four subpanels. Under the proposed changes, one subpanel would cover surgical sciences, obstetrics and gynecology, and child health. A second would cover neuroscience, anesthesia and intensive care, and a third cardiovascular and respiratory medicine. The fourth subpanel would include a range of other medical sciences.

Some heads of medical schools feel that there should be more divisions than this, with each sub-discipline having its own unique rating system scored by specialized experts. But others are equally adamant that creating any subpanels at all would undermine efforts to encourage interdisciplinary research.

The changes are being made in response to the outcome of the 1996 research assessment exercise-carried out by a single panel-under which departments working in separate subjects were judged on a seven-point rating system (Nature Med. 3; 133, 1997). A chief complaint at that time was that placing a broad range of research activities in a single category of 'hospital-based clinical medicine' led to insufficient recognition for some schools, particularly those outside London, that are strong in one subject but weak in another. "We are [hoping] that setting up a system of subpanels is one potential approach to the problem," David Pilsbury of HEFCE told Nature Medicine.
A separate issue raised by the previous assessment exercise that has apparently been resolved is whether clinicians involved in research should have this extra clinical commitment taken into account, given that those in other university disciplines can concentrate purely on teaching and research. There now appears a general consensus that clinical academics should not receive special treatment. "As soon as you say that a subject should be judged by separate criteria, you are implying that the judgements would be less incisive," says Steve Tomlinson, dean of the University of Manchester Medical School, and executive secretary of the council. "It is either distinguished, cost-effective research, or it is not."

But other controversial issues remain to be resolved. For example, if a system of subpanels is set up, should they make their ratings public? And should a new formula be established allowing these individual ratings to be recognized in the overall research ranking of a medical school?

There has also been intense discussion for the past year over the precise division of topics between subpanels. HEFCE's draft proposal to link neuroscience and anesthesia into a single category, for example, is a combination acceptable to some medical schools but not others. Indeed, some schools have already expressed concern that the act of slicing up the assessment of their research in a way which may not correspond to their own overall research strategy represents an unacceptable level of 'micromanagement' of clinical research. But there is also a widespread recognition that selfinterest is at work, and that the strategy favored by any particular medical school is likely to be one that gives it the best chance of obtaining the most money from the funding councils.

HEFCE is organizing a number of seminars around the country later this month at which Pilsbury hopes "a clearer consensus might emerge" on the best way forward. Medical schools and others have been given until October to re spond to HEFCE's proposals, and final guidelines for the 2001 exercise will then be drawn up.

\section{David Dickson, London}

